\title{
"A Cancer in the Minds of Youth?" A Qualitative Study of Problematic Smartphone Use among Undergraduate Students
}

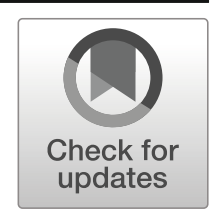

\author{
Zeyang Yang $^{1,2}$ (D) $\cdot$ Kathryn Asbury ${ }^{1} \cdot$ Mark D. Griffiths $^{3}$
}

Published online: 20 December 2019

(C) The Author(s) 2019

\begin{abstract}
There is empirical evidence to suggest that problematic smartphone use (PSU) is associated with mental health problems including anxiety in educational settings. This qualitative study explored attitudes towards - and self-reported impacts of - smartphone use among British young adult students, as well as perceived causes of PSU. Freeresponse written accounts were gathered from 265 British undergraduates at an English university. Open-ended questions were asked about their attitudes towards smartphone use, their reasons for using their smartphones, and what they perceived as the consequences of their smartphone use. Narratives were analyzed using framework analysis and a thematic framework was identified. The three main consequences of PSU described by participants were (1) uncontrolled frequent checking of smartphones, (2) using smartphones late at night, and irrelevant use of smartphones in class. The main reported explanations for PSU were fear of missing messages, boredom in class, poor selfregulation, and external reasons (e.g., boring lectures). Smartphone use was reported to have both positive and negative impacts on young adults' life satisfaction, social relationships, physical health, and study. Many participants reported that they need to develop better self-regulation to address their PSU. Findings suggest that smartphone use can have benefits as well as potentially causing harm among university students. PSU can - in some cases - be understood as reflecting mental well-being issues, poor self-regulation, and social problems.
\end{abstract}

Keywords Student smartphone use · Problematic smartphone use - Smartphone addiction · Perceptions of smartphone use

Smartphones have become ubiquitous in modern society, and young adults appear to be particularly heavy users of them (including the UK where the present study was carried out). The number of smartphone users globally is predicted to reach 2.5 billion in 2019, up

Zeyang Yang

yangzeyangyzy@hotmail.com; yangzeyangyzy@163.com

Extended author information available on the last page of the article 
from 2.1 billion in 2016 (Statista 2019a). In 2018, adult smartphone ownership in the UK was $80 \%$ (Statista 2019b), and was even higher, at $95 \%$, for young adults between the ages of 16 and 24 years (Statista 2019c). In a recent cross-national study of participants in a range of European countries, British young adults reported noticeably high levels of mobile phone dependence, ranking second only to Belgium (Lopez-Fernandez et al. 2017). The reasons for this are unclear; therefore, it is important to investigate why British young adults report such high levels of dependence.

Smartphones have various internet-related functions beyond traditional communication, which has proved highly attractive to young users. Earlier studies found that adolescents were more likely to be "addicted" to smartphones than young adults over the age of 19 years (Haug et al. 2015), and is negatively correlated with age (Van Deursen et al. 2015). However, in a recent study, no clear negative effect of digital-screen use (including smartphones) on wellbeing was found among a very large sample of adolescents (those under 18 years) from Ireland, the USA, and the UK (Orben and Przybylski 2019; Przybylski and Weinstein 2017). The negative effects of screen time on adolescents might be limited, although earlier studies have claimed adolescents are more addicted than young adults (Haug et al. 2015).

Screen use among young individuals appears to differ for adolescents (under 18 years) and young adults (18-29 years) in terms of using social networking sites use (e.g., Twitter) and cell phone ownership (Lenhart et al. 2010). Recent studies among young adults in the UK have found that problematic smartphone use (PSU) has been associated with negative affect (e.g., anxiety), and can be harmful to social interaction (e.g., Chotpitayasunondh and Douglas 2016; Hussain et al. 2017). Given that educational and social contexts are different for adolescents and those in early adulthood, the causes and effects of PSU may differ for the two groups. It is unclear whether smartphone use or PSU changes between school and university. The move to university entails major lifestyle changes and disruption to existing social support networks, as well as increased independence (Yang et al. 2019b). Consequently, it would be useful to further investigate PSU among British young adults.

Many studies have investigated overuse or dependence on smartphones as an addiction (e.g., Bian and Leung 2015; Kwon et al. 2013). A lot of this research has utilized the components model of behavioral addiction which posits that all addictions comprise salience, mood modification, tolerance, withdrawal, conflict, and relapse (Griffiths 2005). However, at present, there are no official criteria for any types of addiction related to mobile phones, smartphones, or the Internet, and the only "technological addiction" (Griffiths 1995) included in the DSM-5 and ICD-11 is gaming disorder (American Psychiatric Association 2013; World Health Organization 2018). Billieux et al. (2015a, b) have cast doubt on use of the term "addiction," pointing out that the specific purpose and context of smartphone use should be carefully considered. It is important to distinguish genuine addiction from problematic use or overuse (Kuss and Griffiths 2012). Therefore, this study applies the term "problematic." It should also be noted that authors such as Kuss and Griffiths (2017) have argued that individuals are no more addicted to smartphones than alcoholics are addicted to bottles and that "smartphone addiction" is an umbrella term that primarily refers to addictions on smartphones rather than addiction to it.

It is likely that the causes and consequences of PSU are complicated. A number of studies in different contexts have found that PSU is associated with poor well-being and other mental health issues (Dayapoglu et al. 2016; Hong et al. 2012; Leung 2008;Long et al. 2016; Yang et al. 2019a). For example, excessive smartphone use has been found to predict anxiety (Lepp et al. 2014). PSU has also been identified as a potential consequence of poor self-regulation 
(Van Deursen et al. 2015). Yang et al. (2019a) assessed self-reported PSU, self-regulation, and well-being issues among undergraduates. They found that self-regulation negatively predicted PSU, and that PSU positively predicted academic anxiety and procrastination. Life satisfaction was also significantly and negatively correlated with PSU. This suggests a need to further investigate the causes, correlates, and consequences of PSU, and qualitative methods are arguably best suited for such exploratory questions.

Most studies investigating PSU have used quantitative data methods. However, a few studies have used qualitative data with interviews of small samples of participants (e.g., Aoki and Downes 2003; Fullwood et al. 2017; Lapointe et al. 2013; Vacaru et al. 2014; Walsh et al. 2008). Fullwood et al. (2017) explored British undergraduates' experiences of, and attitudes towards smartphone use among a sample of 18 participants (four males) in focus groups. Similar to earlier studies (e.g., Aoki and Downes 2003; Lapointe et al. 2013), they found that smartphone use was complex and highly individualized, including functional, informational, interactional, and recreational patterns. Such findings highlight the complexity of understanding smartphone usage and its multifaceted functions. Walsh et al. (2008) conducted focus groups with 32 Australian adolescents and described their general mobile phone usage, identifying perceptions of excessive or addictive mobile phone use, and self-reported symptoms of mobile phone addiction including key consequences found in the components model of addiction including salience, conflict with other activities, and withdrawal (Griffiths 2005). A study among young people (aged 1319 years) in New Zealand also identified addiction-like behaviors among mobile phone users (Vacaru et al. 2014). Therefore, further investigation is needed to examine addictive patterns and consequences of smartphone use among young adults.

A theoretical "pathway" model of problematic mobile phone use (PMPU) was developed by Billieux et al. (2015a, b). They proposed three pathways leading to PMPU: the excessive reassurance pathway, the impulsive-antisocial pathway, and the extraversion pathway. The three pathways were argued to lead to three different types of problematic mobile phone use including an addictive pattern, an antisocial pattern, and a risky pattern. This model highlighted the complexity of PMPU and provided a theoretical framework for studies in this area. For the present study, this theoretical model was applied to guide the qualitative data analysis of the antecedents and patterns of PSU.

A limited number of studies have investigated British university students (young adults) using qualitative methods. These studies attempted to identify smartphone use patterns, reasons, or consequences from small groups of participants (e.g., 32 college students in Aoki and Downes 2003; 18 undergraduates in Fullwood et al. 2017). In order to obtain a more in-depth examination of the potential reasons and consequences for smartphone use or PSU, a much bigger sample is arguably needed. To our knowledge, no studies have collected qualitative data from a relatively large sample size $(>200)$ of British undergraduates, and no studies have presented thematic frameworks for understanding the causes, correlates, and consequences of smartphone use, including PSU, in the UK. Therefore, the present study explored the attitudes, self-perceived reasons, and consequences of British undergraduate students' use of smartphones. In the present study, three specific research questions were examined: (1) How do British undergraduates view their smartphone use? (2) What are the reasons for participants' smartphone use? (3) What do participants see as the consequences of how much they use their smartphones? 


\section{Methods}

\section{Participants}

Participants comprised 303 British undergraduate students (245 females, 57 males, and one non-binary) in a university in Northern England. The participants came from a wide range of departments including archeology, economics, education, engineering, psychology, and music. After excluding the invalid cases who did not respond to any of the open-ended questions (this was one element of a larger study), 265 participants (219 females and 46 males) with valid responses were eligible for analysis. The average age was 20.26 years $(\mathrm{SD}=3.40)$.

\section{Materials}

There were five open-ended questions that were included as part of a larger survey: (1) Could you describe how you use your smartphone during class or other learning periods? (2) Could you talk a bit about the others' use of smartphones as you have observed it, either in their daily lives or during classes and other learning periods? (3) What factors motivate you or your friends to use smartphones? (4) What are the possible impacts, if any, of smartphone use to you or your friends? and (5) Do you think you use your smartphone too much? If yes, why? What problems do this cause for you? The questions were developed according to the research questions of the present study, including students' views on their own and others' smartphone usage (questions 1 and 2), the reasons (question 3), and the consequences (question 4). The fifth question was added to ask the participants to evaluate their smartphone use. The participant's level of PSU was also assessed using the smartphone addiction scale-short version (SAS-SV; Kwon et al. 2013). The SAS-SV scores are the total scores on a six-point 10-item Likert scale (potential range of 10-60). Example items include "Sometimes I use my smartphone for longer than I intend to" and "I have missed planned work due to smartphone use." The developers of the SAS-SV set the thresholds as 33 (for females) and 31 (for males) for being at risk of addiction, based on clinical psychologists' consultation results. The SAS-SV, with its addiction thresholds (cut-off points), was adopted in order to identify the potential problematic users who perceive their PSU at higher levels in a self-reported scale. The present study used a mixed-methods design because it allows for quantitative and qualitative data triangulation and helps to gain better insight and new information compared with single-method use (Creswell and Plano Clark 2017). However, the major findings of the present study were obtained from the qualitative data.

\section{Procedure}

The first author distributed the paper questionnaires around the university campus and during breaks in lectures, where staff permission had been given. As part of this paper-based data collection process, the first author introduced the study to potential participants before distributing the surveys. The students read the consent information before deciding whether to participate. At the same time, an online version of the questionnaire, using Qualtrics, was distributed to undergraduate administrators or departmental receptionists via email. In addition, a QR code for the online questionnaire, together with information about the study, was printed and posted in public spaces around campus. Participants completed the open-ended questions 
at the end of a series of closed items regarding problematic smartphone use, academic emotions, and well-being.

\section{Data Analysis}

The framework analytic approach (Ritchie and Spencer 1994; Gale et al. 2013) was used for this qualitative data analysis as this is particularly well suited to the organization and interpretation of large qualitative datasets. Five steps were applied: familiarization, identifying a thematic framework, indexing, charting, and mapping. Since computerassisted qualitative data analysis software (CAQDAS) is useful to increase the efficiency of framework analysis (Gale et al. 2013), all analyses were conducted using the NVivo version 11. In the first step, the first author reads all transcripts and became familiar with the main ideas and thoughts of the interviewees. In the second step, in order to identify a thematic framework, coding was conducted in several transcripts before a draft thematic framework was identified. However, since the thematic framework cannot be finally confirmed before the last transcript is coded (Gale et al. 2013), coding and framework modification proceeded simultaneously until the last transcript was coded in this step. In the third step, the thematic framework was applied to all transcripts in order to organize all data for interpretation. In the fourth step, all data were charted into a matrix in NVivo with all cases in rows and all themes in columns. Finally, data were interpreted according to the thematic framework.

\section{Results}

Table 1 outlines the thematic framework developed from the data generated by the participants. The themes identified included smartphone usage, antecedents, impacts, and attitudes towards smartphone use. Smartphone use was classified as either non-problematic use (general use and study) or problematic use. Problematic use was operationally defined based on two aspects: SAS-SV scores and descriptions of problematic mobile phone use patterns in the pathway model by Billieux et al. (2015a, b). For example, if the participant described their inappropriate use in class (second pathway in the model), or addiction-like behaviors (first pathway in the model), the narration was coded as problematic. If the SAS-SV score was over thresholds for problematic use (33 females and 31 males), the participant's narrations might be considered to be associated with PSU depending upon the context mentioned. In response to the question "Do you think you use your smartphone too much?" $(N=265)$, more than half $(57 \%)$ reported that they did. However, it was found that some problematic users (those who exceeded the SAS-SV threshold) did not describe their use as problematic $(18.30 \%, 20$ out of 109), and some with low scores on the SAS-SV $(55.10 \%, 86$ out of 156) reported concerns about their smartphone use.

\section{What Do UK Undergraduates Use Their Smartphones for?}

Both general use and problematic use of smartphone were reported by participants. Smartphones were frequently reported as being essential tools for daily life, including being required for contacting people, using social media, information searching, and assisting with travel. For instance: 
Table 1 Thematic framework for smartphone use among British young adults $(N=265)$

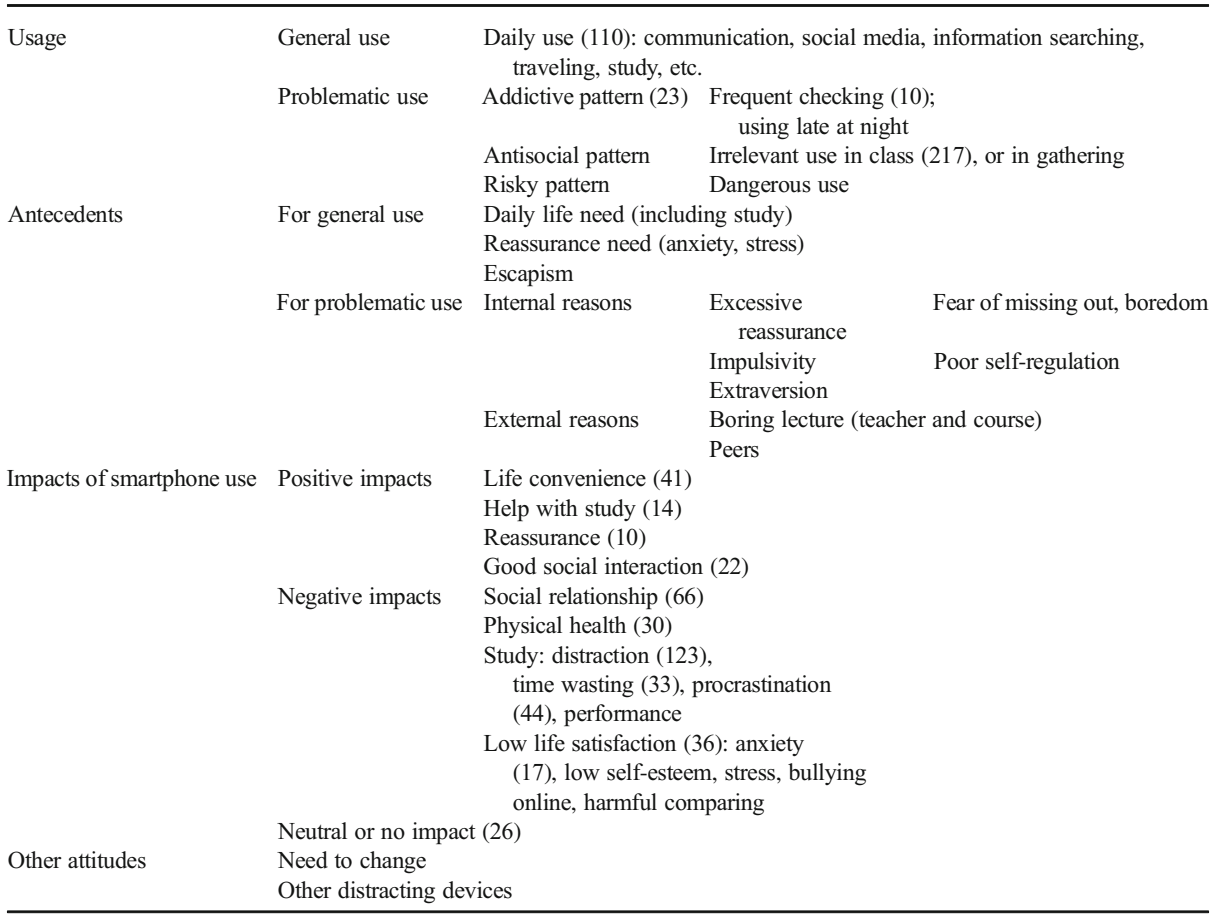

The numbers of participants who made a comment fitting each of the themes is shown in brackets. Numbers lower than 10 participants are not shown

"Ease of access to multiple apps and platforms that aid daily lives such as calendars and ease of communication via emails, sms and social media" (Female, 18, SAS-SV score 21).

Smartphones were also frequently reported as tools for study. For example:

"I usually use it for looking things up that I don't understand, which relates to what we are learning about" (Female, 20, SAS-SV score: 26).

"To answer class polls on Menti and to find more information on topics that are interesting or that I don't understand" (Female, 20, SAS-SV score: 30).

However, as well as general use and for study purposes, the features of PSU described in Billieux et al.'s (2015a, b) model were mentioned by participants. Twelve participants reported that they used smartphones too much or in an addictive way using the words "addiction," "addictive," "dependent," or similar descriptions. For example:

"I don't use it (in class). Smartphones are great, but they have become a cancer in the minds of the youth. They use them as much as they breathe" (Male, 20, SAS-SV: 12).

"I use my phone too much as I have a considerable capacity for needless distraction and leads to an almost addictive relationship with the instantly gratifying processes of messaging, liking, scrolling etc." (Male, 18, SAS-SV: 49). 
"I am probably addicted to my phone in the way that I can now be so easily distracted to go on my phone, especially if I am talking with a boy and want to check to see if he has contacted me or read my messages. This then makes me annoyed at myself as I have wasted time that I needed for studying and not used my time efficiently" (Female, 22. SAS-SV: 36).

However, one participant was not sure whether it should be considered addictive:

"People often have a quick look in lectures, and you see people sitting around in the library on their phones. What with the multi-purpose aspect of smartphones, you don't know if people are using it to communicate with friends, look at the news or just browse about on shopping sites. People do seem pretty wedded to their phones but I'm not aware of too many people who I'd describe as straight-up 'addicted' to their phones" (Female, 22).

Irrelevant use of smartphones in learning contexts or antisocial use was frequently mentioned. For instance:

"Although I sometimes use it to look up material relevent to the class, I usually use it for social media to distract myself" (Male, 19, SAS-SV: 36).

\section{Antecedents for PSU}

Students reported antecedents for both general smartphone use and PSU. Since the comments on the antecedents for general use are largely similar to those described above, this section specifically focuses on perceived antecedents for PSU. Both internal and external antecedents for PSU were reported. Internal reasons included excessive need for reassurance (fear of missing out, boredom), impulsivity (poor self-regulation), and extraversion, which align with the three pathways (excessive reassurance, impulsivity, and extraversion) in the model by Billieux et al. (2015a, b). For example:

"It is addictive- don't want to miss out" (Female, 19, SAS-SV: 36).

"I feel like I can't put it down because I constantly want to check what is happening on my phone" (Female, 18 SAS-SV: 55).

Anxiety and stress were mentioned as antecedents for either smartphone use or PSU. These fit best under the theme of "reassurance need." Since the participants did not clearly note the situations of smartphone use (either in class or in daily life), it appears difficult to judge whether they are antecedents for PSU or general use. External reasons offered for inappropriate smartphone use included boring lectures or peers doing the same thing:

"I would only go on my phone if I found that the class was a waste of time or if I urgently had to reply to someone. When I am surrounded people who are also procrastinating, I find it easy to do myself without feeling guilty" (Female, 22, SAS-SV: 36).

Escapism was reported because the participants might use phones to avoid social interaction. It might be understood as a reason for PSU or the anti-social pattern of PSU. For example:

"As an introvert, my smartphone becomes a defence or somewhere to hide when I am anxious in public" (Female, 25; SAS-SV: 32). 
"Too easy to disappear into phone instead of interacting face to face" (Female, 18, SASSV: 32).

"To escape (through apps but mostly listening to music) as a social crutch" (Male, 21, SAS-SV: 42).

\section{Impacts of Smartphone Use}

Both positive and negative impacts of smartphone use were reported. Positive impacts include life convenience, help with study, and reassurance when anxious or stressed. These positive impacts might also be found in the comments about the usage of smartphones. For example:

"Convenience - in terms of apps that help organise and track things, connecting with friends, ease of communication. Listening to music. Being able to find out information at the click of a button" (Female, 21, SAS-SV score: 27).

"I usually use it for looking things up and that I don't understand, which relates to what we are learning about" (Female, 20, SAS-SV score: 26).

"Stress, anxiety and a release from stress and anxiety... when I don't look at my phone all day, I feel a release but also stress because I know I will have missed loads of important emails and messages" (Female, 22, SAS-SV score: 32).

Negative impacts included worse social, physical health issues, problems with study (distraction, time wasting, and procrastination) and low life satisfaction. For example:

"I think people are becoming detached from reality. It's uncommon now for a group of people to go out and not look at their phones.I find this really irritating" (Female, 21, SAS-SV score: 14).

"Sometimes after long periods, eyes start to ache and get tired" (Female, 20, SAS-SV score: 44).

"For me it's non existent. However, most of my friends will put off work so they can scroll through social media and waste hours of their lives looking at other people waste theirs" (Male, 20, SAS-SV score: 12).

Harmful interpersonal comparing and bullying online were mentioned by some participants. In a comment on bullying, privacy was also mentioned:

"Becoming reliant and unable to live without. Easy access to social media allows you to see what other people are doing and compare yourself to them socially and academically. This can affect ones self confidence and make them feel inadequate/unhappy" (Male, 20, SAS-SV score: 20).

"Bullying online, people knowing all of your business, gossiping" (Female, 19, SAS-SV score: 45$)$. 


\title{
Other Attitudes Towards Smartphone Use
}

The positive and negative attitudes were indicated by the comments concerning impacts as noted above. More specifically, many participants reported that they used smartphones problematically and need to reduce their usage.

"Yes, I do (use it too much). I don't know exactly why and I do think I should try and use my phone less" (Female, 19, SAS-SV score: 35).

One student reported that smartphone was not the only distractor since they may also turn to other devices such as laptops during lectures for inappropriate use. It is important to note that smartphones are not the only devices to distract students from their tasks. When students cannot control their behavior, they may lose concentration and use smartphone, laptops, or other devices for distraction. This suggests the importance of self-regulation in their lives.

\begin{abstract}
"In class I see a lot of people using laptops to check social media - this then looks to the teachers like they are taking notes. However, this seems to be a way of accessing smartphone features and apps through another device - if it were allowed or not considered rude to be on smartphones in class these would probably be used instead. In terms of daily lives, I rarely seem to see people glued to their phone when they are in social groups, but when they are alone in a public setting people (perhaps under the age of 40) will be on their smartphone" (Female, 21, SAS-SV score: 30).
\end{abstract}

\section{Discussion}

The present qualitative study explored four main over-arching themes or areas: attitudes towards smartphone use (problematic and non-problematic), antecedents of smartphone use, and impacts of smartphone use. Overall, the results suggested that the smartphone use of British university students should be discussed from both positive and negative perspectives. Smartphones were reported as important tools in the participants' daily life. General use of smartphones, especially for academic study, cannot be ignored, although in some contexts the use of smartphones can be problematic. Reasons reported for PSU included fear of missing out, need for reassurance, poor self-regulation (poor self-control), and sensation seeking, which reflect the three pathways into PSU proposed by Billieux et al. (2015a, b): excessive reassurance, impulsivity, and extraversion. The participants' own views were not always in line with their SAS-SV scores. Approximately one-fifth (18.3\%) of the high scorers (over the addiction threshold) did not describe their smartphone use as problematic, while $55.10 \%$ of the low scorers reported PSU in their responses. In line with Billieux et al. (2015a, b), this indicates the potential limitation of diagnosing PSU or smartphone addiction using symptom-based approaches only.

Poor course design and boring lecturers were additional reasons given for PSU. Furthermore, several symptoms of PSU were reported including frequent checking, using smartphones late at night, inappropriate use in class, and use on the road. These consequences 
can be discussed in the context of three patterns proposed by Billieux et al. (i.e., an addictive pattern, an antisocial pattern, and a risky pattern). Beyond the pathway model, the findings of the present study highlight the external and/or environmental impacts on PSU (e.g., boring lectures). Both positive and negative impacts of smartphone use were reported. On the positive side, smartphone use was seen as contributing to life convenience, helping with academic study, and improving social relationships. On the negative side, PSU was seen as negatively affecting social relationships, academic study (distraction, procrastination, test anxiety), physical health, and privacy. Participants regarded smartphones as necessary tools in daily life. However, many of them said they needed to change their patterns of use (i.e., reducing time spent on smartphones) by improving their self-regulation or setting goals.

The present qualitative study identified similar themes to other empirical qualitative studies concerning smartphone/mobile phone use. In line with the findings of Fullwood et al. (2017), the present study confirmed that smartphones were a "social crutch" and sometimes embarrassing in social situations. Similar to previous studies (Aoki and Downes 2003; Lapointe et al. 2013; Walsh et al. 2008), the study here identified different facets of smartphone use including communication, dependence, and anti-social usage. As with several previous quantitative and qualitative studies (Lapointe et al. 2013; Vacaru et al. 2014; Yang et al. 2019a, b), both negative and positive impacts of smartphones on social activities, learning, and/or well-being were identified in the present study. However, there were also themes that appeared in other studies that were not found here including financial incentives (Aoki and Downes 2003), use in emergency situations (Walsh et al. 2008), and fear of rejection (Lapointe et al. 2013). Furthermore, there were several aspects identified in the present study that have not been reported in any previous studies including escapism, harmful interpersonal comparisons, and online bullying.

The findings reported here have potential implications for understanding of smartphone use or wider screen use among UK undergraduate students. It appears necessary to consider both positive and negative effects of screen use, instead of simply over-pathologizing students' overuse of smartphones. On one hand, the thematic framework indicated that smartphone use among university students can assist their learning and improve life satisfaction. The benefits of new technology and new smartphone-based learning applications cannot be ignored. On the other hand, negative impacts of smartphone use (either generalized antisocial PSU in class or specific PSU on social media) cannot be ignored. It appears that PSU is not a simple behavioral problem but in some cases may reflect deeper problems underlying mental wellbeing, self-regulation, and social communication. Further investigation is needed focusing on undergraduates' well-being related to smartphone use (or screen use more generally), especially given how quickly the smartphone industry is evolving.

In terms of "addiction," it remains unclear whether the students in the present study were really addicted to the smartphone functions or the phone itself as a "social crutch." No participants endorsed all six criteria in the components model of behavioral addiction (Griffiths 2005). It appears probable that the real concern underlying PSU or smartphone addiction may be the problems related to either a specific function (e.g., social media use disorder, gaming disorder) or social communication problems (e.g., social phobia, escapism) (Kuss and Griffiths 2017). Therefore, as argued by one participant, all other electronic devices (e.g., tablets, laptops) might replace smartphones as a tool or a toy. Therefore, the findings of the present study suggest that scholarly concern should not only focus on the "addiction-or-not?" question but also the psychological and social problems that are consequential to PSU. 
It should be noted that there are several limitations of the present study. The possibility of socially desirable answers could be one limitation. Furthermore, it is possible that this crosssectional study with a single wave of data collection misses changes or developments in individuals' smartphone use. Participants might modify their behaviors on smartphones during their university life because some participants indicated that they needed to change the problematic aspects of their smartphone use. Consequently, future studies should comprise longitudinal and experimental elements related to undergraduate students' or young adults' use of smartphones. Tracking smartphone use longitudinally throughout university life would be one line of investigation to follow. In order to avoid social desirable responses, future studies could also investigate PSU or screen use using online tracking data rather than survey data as has been done in research on gambling and gaming.

Authors' Contribution Zeyang Yang and Kathryn Asbury designed the study. Zeyang Yang conducted data collection and analysis. Zeyang Yang carried out the main bulk of the manuscript writing and literature review. Kathryn Asbury participated in checking methods and results and supporting Zeyang Yang during the data collection and analysis. Kathryn Asbury and Mark D. Griffiths acted in an editorial role when it came to the writing up of the research study. All authors read and approved the final manuscript.

\section{Compliance with Ethical Standards}

Conflict of Interest The authors declare that they have no conflict of interest.

Ethical Approval This study has been approved by the Ethics Committee of the Department of Education at the University of York. All procedures performed in this study involving human participants were in accordance with the ethical standards of University's Research Ethics Board and with the 1975 Helsinki Declaration.

Informed Consent Informed consent was obtained from all participants.

Open Access This article is licensed under a Creative Commons Attribution 4.0 International License, which permits use, sharing, adaptation, distribution and reproduction in any medium or format, as long as you give appropriate credit to the original author(s) and the source, provide a link to the Creative Commons licence, and indicate if changes were made. The images or other third party material in this article are included in the article's Creative Commons licence, unless indicated otherwise in a credit line to the material. If material is not included in the article's Creative Commons licence and your intended use is not permitted by statutory regulation or exceeds the permitted use, you will need to obtain permission directly from the copyright holder. To view a copy of this licence, visit http://creativecommons.org/licenses/by/4.0/.

\section{References}

American Psychiatric Association. (2013). Diagnostic and statistical manual of mental disorders: DSM-5. Arlington: American Psychiatric Publishing.

Aoki, K., \& Downes, E. J. (2003). An analysis of young people's use of and attitudes toward cell phones. Telematics and Informatics, 20(4), 349-364.

Bian, M., \& Leung, L. (2015). Linking loneliness, shyness, smartphone addiction symptoms, and patterns of smartphone use to social capital. Social Science Computer Review, 33(1), 61-79.

Billieux, J., Maurage, P., Lopez-Fernandez, O., Kuss, D. J., \& Griffiths, M. D. (2015a). Can disordered mobile phone use be considered a behavioral addiction? An update on current evidence and a comprehensive model for future research. Current Addiction Reports, 2(2), 156-162.

Billieux, J., Philippot, P., Schmid, C., Maurage, P., De Mol, J., \& Van der Linden, M. (2015b). Is dysfunctional use of the mobile phone a behavioural addiction? Confronting symptom-based versus process-based approaches. Clinical Psychology \& Psychotherapy, 22(5), 460-468. 
Chotpitayasunondh, V., \& Douglas, K. M. (2016). How "phubbing” becomes the norm: The antecedents and consequences of snubbing via smartphone. Computers in Human Behaviour, 63, 9-18.

Creswell, J. W., \& Plano Clark, V. L. (2017). Designing and conducting mixed methods research (Third ed.). Los Angeles: Sage.

Dayapoglu, N., Kavurmaci, M., \& Karaman, S. (2016). The relationship between the problematic mobile phone use and life satisfaction, loneliness, and academic performance in nursing students. International Journal of Caring Sciences, 9(2), 647-652.

Fullwood, C., Quinn, S., Kaye, L. K., \& Redding, C. (2017). My virtual friend: a qualitative analysis of the attitudes and experiences of smartphone users: implications for smartphone attachment. Computers in Human Behavior, 75, 347-355.

Gale, N. K., Heath, G., Cameron, E., Rashid, S., \& Redwood, S. (2013). Using the framework method for the analysis of qualitative data in multi-disciplinary health research. BMC Medical Research Methodology, 13, 117.

Griffiths, M. D. (1995). Technological addictions. Clinical Psychology Forum, 76, 14-19.

Griffiths, M. (2005). A 'components' model of addiction within a biopsychosocial framework. Journal of Substance Use, 10(4), 191-197.

Haug, S., Castro, R. P., Kwon, M., Filler, A., Kowatsch, T., \& Schaub, M. P. (2015). Smartphone use and smartphone addiction among young people in Switzerland. Journal of Behavioral Addictions, 4(4), 299307.

Hong, F.-Y., Chiu, S.-I., \& Huang, D.-H. (2012). A model of the relationship between psychological characteristics, mobile phone addiction and use of mobile phones by Taiwanese university female students. Computers in Human Behavior, 28(6), 2152-2159.

Hussain, Z., Griffiths, M. D., \& Sheffield, D. (2017). An investigation into problematic smartphone use: the role of narcissism, anxiety, and personality factors. Journal of Behavioral Addictions, 6(3), 378-386.

Kuss, D. J., \& Griffiths, M. D. (2012). Internet gaming addiction: a systematic review of empirical research. International Journal of Mental Health and Addiction, 10(2), 278-296.

Kuss, D. J., \& Griffiths, M. D. (2017). Social networking sites and addiction: ten lessons learned. International Journal of Environmental Research and Public Health, 14, 311.

Kwon, M., Kim, D. J., Cho, H., \& Yang, S. (2013). The smartphone addiction scale: development and validation of a short version for adolescents. PLoS One, 8(12), e83558.

Lapointe, L., Boudreau-Pinsonneault, C., \& Vaghefi, I. (2013). Is smartphone usage truly smart? A qualitative investigation of IT addictive behaviors. In System sciences (HICSS), 2013 46th Hawaii international conference on system sciences (pp. 1063-1072). Grand Wailea: IEEE.

Lenhart, A., Purcell, K., Smith, A., \& Zickuhr, K. (2010). Social media \& mobile internet use among teens and young adults. Millennials. Pew Internet \& American life Project. Retrieved July 21 2019, from: https://files. eric.ed.gov/fulltext/ED525056.pdf

Lepp, A., Barkley, J. E., \& Karpinski, A. C. (2014). The relationship between cell phone use, academic performance, anxiety, and satisfaction with life in college students. Computers in Human Behavior, 31(1), $343-350$.

Leung, L. (2008). Linking psychological attributes to addiction and improper use of the mobile phone among adolescents in Hong Kong. Journal of Children and Media, 2(2), 93-113.

Long, J., Liu, T. Q., Liao, Y. H., Qi, C., He, H. Y., Chen, S. B., \& Billieux, J. (2016). Prevalence and correlates of problematic smartphone use in a large random sample of Chinese undergraduates. BMC Psychiatry, 16(1), 408.

Lopez-Fernandez, O., Kuss, D. J., Romo, L., Morvan, Y., Kern, L., Graziani, P., et al. (2017). Self-reported dependence on mobile phones in young adults: a European cross-cultural empirical survey. Journal of Behavioral Addictions, 6(2), 168-177.

Orben, A., \& Przybylski, A. K. (2019). Screens, teens, and psychological well-being: evidence from three timeuse-diary studies. Psychological Science, 30(5), 682-696. https://doi.org/10.1177/0956797619830329.

Przybylski, A. K., \& Weinstein, N. (2017). A large-scale test of the goldilocks hypothesis: quantifying the relations between digital-screen use and the mental well-being of adolescents. Psychological Science, 28(2), $204-215$.

Ritchie, J., \& Spencer, L. (1994). Qualitative data analysis for applied policy research. In A. Alan Bryman \& B. Bob Burgess (Eds.), Analyzing qualitative data (pp. 173-194). London: Taylor \& Francis.

Statista (2019a). Number of smartphone users worldwide from 2014 to 2020 (in billions). Retrieved June 1, 2019, from: https://www.statista.com/statistics/330695/number-of-smartphone-users-worldwide/.

Statista (2019b). Smartphone adoption in the United Kingdom (UK) from 2011 to 2018. Retrieved June 1, 2019, from https://www.statista.com/statistics/271460/smartphone-adoption-in-the-united-kingdom-uk/ .

Statista (2019c). UK: smartphone ownership by age from 2012-2018. Retrieved June 1, 2019, from https://www. statista.com/statistics/271851/smartphone-owners-in-the-united-kingdom-uk-by-age/ 
Vacaru, M. A., Shepherd, R. M., \& Sheridan, J. (2014). New Zealand youth and their relationships with mobile phone technology. International Journal of Mental Health and Addiction, 12, 572-584.

Van Deursen, A. J., Bolle, C. L., Hegner, S. M., \& Kommers, P. A. (2015). Modeling habitual and addictive smartphone behavior: the role of smartphone usage types, emotional intelligence, social stress, self-regulation, age, and gender. Computers in Human Behavior, 45, 411-420.

Walsh, S. P., White, K. M., \& Young, R. M. (2008). Over-connected? A qualitative exploration of the relationship between Australian youth and their mobile phones. Journal of Adolescence, 31(1), 77-92.

World Health Organization (2018). ICD-11 for mortality and morbidity statistics. Retrieved June 1, 2019, from https://icd.who.int/browse11/1-m/en\#/http\%3a\%2f\%2fid.who.int\%2ficd\%2fentity\%2f1448597234.

Yang, Z., Asbury, K., \& Griffiths, M. D. (2019a). An exploration of problematic smartphone use among Chinese university students: associations with academic anxiety, academic procrastination, self-regulation and subjective wellbeing. International Journal of Mental Health and Addiction, 17(3), 596-614.

Yang, Z., Asbury, K., \& Griffiths, M. D. (2019b). Do Chinese and British university students use smartphones differently? A cross-cultural mixed methods study. International Journal of Mental Health and Addiction, 17(3), 644-657.

Publisher's Note Springer Nature remains neutral with regard to jurisdictional claims in published maps and institutional affiliations.

\section{Affiliations}

\section{Zeyang Yang ${ }^{1,2} \cdot$ Kathryn Asbury ${ }^{1} \cdot$ Mark D. Griffiths $^{3}$}

1 Psychology in Education Research Centre, Department of Education, University of York, York, UK

2 Faculty of Education, University of Macau, Macau, China

3 International Gaming Research Unit, Psychology Department, Nottingham Trent University, Nottingham, UK 